\title{
Developing a pediatric neurosurgical training model
}

\author{
Claudia L. Craven, MSc, MRCS, ${ }^{1}$ Martyn Cooke, MSc, ${ }^{2}$ Clare Rangeley, BA, ${ }^{2}$ \\ Samuel J. M. M. Alberti, PhD, ${ }^{2}$ and Mary Murphy, MD, FRCS(SN) ${ }^{1}$ \\ 'Victor Horsley Department of Neurosurgery, National Hospital for Neurology and Neurosurgery, Queen Square; and ${ }^{2}$ Museums \\ Conservation Unit, The Royal College of Surgeons of England, London, United Kingdom
}

OBJECTIVE One of the greatest challenges of pediatric neurosurgery training is balancing the training needs of the trainee against patient safety and parental expectation. The traditional "see one, do one, teach one" approach to training is no longer acceptable in pediatric neurosurgery. The authors have developed the baby Modeled Anatomical Replica for Training Young Neurosurgeons (babyMARTYN). The development of this new training model is described, its feasibility as a training tool is tested, and a new approach of integrating simulation into day-to-day training is suggested.

METHODS In part 1 (development), a prototype skull was developed using novel model-making methods. In part 2 (validation), 18 trainee neurosurgeons (at various stages in training) performed the following 4 different procedures: 1) evacuation of a posterior fossa hematoma; 2) pterional craniotomy; 3) tapping of the fontanelle to obtain a CSF specimen; and 4) external ventricular drain insertion. Completion of the procedural stages (scored using a curriculum-based checklist) was used to test the feasibility of babyMARTYN as a training tool. Likert scale-based questionnaires were used to assess the model for face and content validity. Training benefit was assessed using pre- and posttraining ratings on the Physician Performance Diagnostic Inventory Scale (PPDIS). To determine the significance of improvement in median PPDIS score, the Wilcoxon matched-pairs signed-rank test was performed.

RESULTS In part 1 (development), the model was successfully developed with good fidelity. In part 2 (validation), the validation data demonstrated feasibility, face, and content validity. The PPDIS score significantly increased for all groups after babyMARTYN training, thereby indicating a potential future role for babyMARTYN in the training of pediatric neurosurgeons.

CONCLUSIONS This recent collaborative neurosurgical development by the Royal College of Surgeons of England is designed to supplement current neurosurgical training. High-fidelity, portable, operation-specific models enable preoperative planning and have the potential to be used in an operating room environment prior to novel operations. A "see one, simulate one, do one" approach for pediatric neurosurgical training using babyMARTYN is suggested.

https://thejns.org/doi/abs/10.3171/2017.8.PEDS17287

KEY WORDS simulation; pediatric; training; model; manikin; education; neurosurgery; surgical technique

$\mathrm{T}$ HE traditional "see one, do one, teach one" approach to training is becoming increasingly outdated, and the public no longer views it as an acceptable training method. ${ }^{8}$ The safety and training benefits of simulation are becoming more recognized, with various models being developed for adults in the field of neurosurgery. ${ }^{1-3,5-7,12}$ The value of simulation training is recognized by the Joint Committee of Surgical Training, including the simulationbased assessments of the Intercollegiate Surgical Curricu- lum Programme, which is the main curriculum in the United Kingdom (UK) (https://www.iscp.ac.uk/Default.aspx). ${ }^{8}$

One field that would benefit particularly from a revolution of simulation training is pediatric surgery and, in particular, pediatric neurosurgery. Pediatric neurosurgical training presents a challenge, especially when it comes to balancing the safety of the child and providing adequate operative exposure to trainees. In many regions, pediatric neurosurgery is centralized, which enables specialist care

ABBREVIATIONS babyMARTYN = baby Modeled Anatomical Replica for Training Young Neurosurgeons; $C C T=$ certificate of completion of training; EVD = external ventricular drain; PPDIS = Physician Performance Diagnostic Inventory Scale; UK = United Kingdom.

SUBMITTED May 24, 2017. ACCEPTED August 1, 2017.

INCLUDE WHEN CITING Published online December 22, 2017; DOI: 10.3171/2017.8.PEDS17287. 
for children to be focused and concentrated in specialist units and thus reduces exposure for the trainee. It is typical for neurosurgery trainees in the UK to begin their placements in such pediatric units late in their training, after having developed the basic foundations of operative skills. Even in units in which early trainee exposure to pediatric neurosurgery is possible, the care remains heavily consultant driven. Clearly this ensures the safety of the child; however, the combination of these factors often means that it takes longer to develop the required skills to safely perform pediatric neurosurgery. There inevitably comes a point when a surgeon has to perform an operation for the first time. Ultimately, all British trainees need to be competent in emergency pediatric neurosurgery before completion of their training.

Training challenges could be addressed by the application of a high-fidelity training model to enable preoperative rehearsal. There is currently no existing infant model that enables a wide range of procedures to be performed. There is a paucity of pediatric neurosurgical synthetic training models and particularly infant models, an area in which simulation is possibly needed the most.

We developed a high-fidelity simulation model, the baby Modeled Anatomical Replica for Training Young Neurosurgeons (babyMARTYN). BabyMARTYN was initially designed to rehearse 4 key procedures in infants: 1) evacuation of a posterior fossa lesion (hemorrhage or tumor); 2) pterional craniotomy; 3) tapping of the fontanelle to obtain a CSF specimen; and 4) external ventricular drain (EVD) insertion. We describe the development of babyMARTYN as a training model for infant neurosurgery, and we used these 4 procedures to test its feasibility and validity (face, construct, and content validity) as a training tool. Finally, we suggest how such training models can be integrated into the current training program.

\section{Methods \\ Model Development}

A 3D print was made from a scan of an 18-monthold infant that combined the features of an infant skull in the Royal College of Surgeons of England museum collection (Fig. 1A). This 3D print was then used as the template mold for simplified prototype skulls (Fig. 1B). The babyMARTYN brain was molded by an artist who specializes in anatomical models and used the operative images and cadaveric specimens (age 18 months) as references. Detailed anatomical features were molded and cast in the appropriate materials, with the bone being set in a polyurethane composite, the brain (with CSF ventricle spaces) in a gelatin composite, and the dura mater in moldable plastic (Fig. 1C). The model was designed to enable 4 major procedures to be performed.

For each of the 4 procedures, key anatomical details were identified and included. 1) For evacuation of a posterior fossa lesion (hemorrhage or tumor), the cerebellum (complete with folia and vermis) and access to the fourth ventricle were included. A large, firm gelatin intracranial hemorrhage was incorporated into the vermis (Fig. 1C). The medulla and spinal cord, including the $\mathrm{C}-1$ vertebra, were included in the model. 2) For pterional craniotomy,
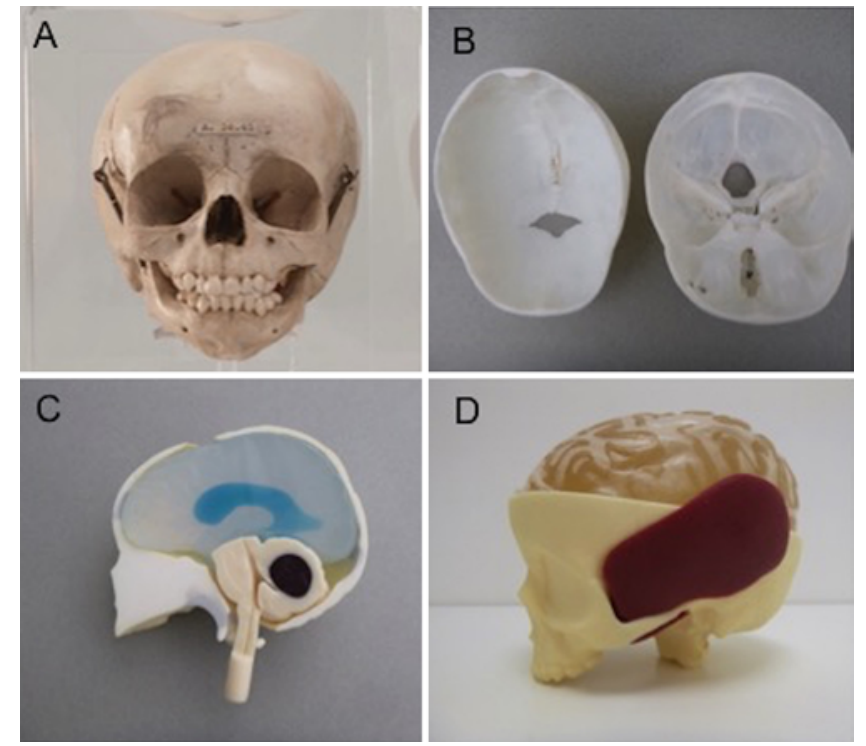

FIG. 1. Components of babyMARTYN. A: An infant skull (Specimen RCSOM/A 14.41) underwent 3D scanning. CMuseums at the Royal College of Surgeons. B: This 3D print was used as the template mold for simplified prototype skulls and cast in polyurethane resin. C: Midsagittal view of the model skull containing the cerebrum, cerebellum, fluid-filled ventricles, medulla, spinal cord, intracranial hemorrhage, and vermis. D: The model with the silicone-based temporalis muscle. The vault of the cranium was removed from this model to show the underlying sulci and gyri. Figure is available in color online only.

a silicone-based temporalis muscle was firmly adhered to the skull with accurate underlying sulci and gyri, including the sylvian fissure (Fig. 1D). 3) To tap the fontanelle to obtain a CSF specimen, the skull has an anterior fontanelle with a thin layer of underlying dura mater under which thin paraffin oil (to replicate CSF) was present. 4) For EVD insertion, the coronal suture and orbits were included as bony landmarks. The ventricles were filled with thin paraffin oil to replicate CSF and injected into the ventricular spaces via the fontanelle.

\section{Feasibility Trial}

All 4 operations were performed by 13 trainees (Table 1). To test the model's feasibility to simulate each operative stage, a checklist was created (and adapted for pediatrics) based on the current Intercollegiate Surgical Curriculum Programme syllabus guidance (Table 2). ${ }^{2}$ Each trainee was filmed performing each procedure to test the model's potential as an assessment tool and ability to fulfill each of the checklist criteria.

\section{Physician Performance Diagnostic Inventory Scale}

Each trainee self-rated his or her pretraining performance on the Physician Performance Diagnostic Inventory Scale (PPDIS) as "unsatisfactory," "early learner," "competent," "proficient," or "expert" (scores 1-5, respectively). This was repeated after training on babyMARTYN to determine if babyMARTYN had a positive impact on PPDIS. To determine the significance of any improvement in median PPDIS, the Wilcoxon matched-pairs signedrank test was performed. A $p$ value $<0.05$ was considered 
TABLE 1. Participants in the validation study

\begin{tabular}{lc}
\hline \multicolumn{1}{c}{ Level of Training } & No. of Participants \\
\hline Medical student (total novice) & 1 \\
\hline Foundation year (first 2 years postgraduation) & 2 \\
\hline ST1 (junior resident) & 1 \\
\hline ST2 (junior resident) & 2 \\
\hline ST3 (junior resident) & 2 \\
\hline ST4 (senior resident) & 0 \\
\hline ST5 (senior resident) & 2 \\
\hline ST6 (senior resident) & 1 \\
\hline Post-CCT & 1 \\
\hline Consultant neurosurgeon & 1 \\
\hline
\end{tabular}

$\mathrm{ST}=$ specialist trainee.

significant. Linear regression analysis was used to determine if there was correlation between PPDIS and training level and if this correlation was altered after training on babyMARTYN. All statistical tests were completed on GraphPad Prism v6.0.

\section{Face and Content Validity}

Anonymous feedback was collected using a standardized questionnaire with the 5-point Likert scale and analyzed by blinded investigators. Face validity data (specifically tactile and visual realism) were prospectively collected using Likert scale ratings $(1=$ unrealistic, $5=$ highly realistic). Content validity was tested using a questionnaire by asking, "Was the model useful?" and "Would you recommend the model as a training tool?"

\section{Results}

\section{Feasibility Trial}

Four operations were performed on babyMARTYN by 13 individuals (Fig. 2). To determine the feasibility of babyMARTYN for use in training, its ability to test various components of a checklist based on the current curriculum was assessed (Table 2). 1) For evacuation of a posterior fossa lesion (hemorrhage or tumor) including decompression of the C-1 arch, 11 of 14 stages were completed but the final 3 stages (hemostasis, skin closure, and wound repair) could not be assessed on this prototype because it is currently skinless (Fig. 2A and B). 2) For pterional craniotomy, 7 of 9 key stages were feasible and only Stage 6 (hemostasis) and Stage 9 (skin closure) could not be assessed on this model (Fig. 2C and D). 3) For tapping of the fontanelle to obtain a CSF specimen, all 6 stages were feasibly simulated (Fig. 2E). 4) For EVD insertion, 9 of 11 stages were completed, but Stage 9 (skin closure) could not be assessed, and Stage 8 could only be partially achieved (because a small amount of negative pressure from a syringe was required to encourage CSF flow; Fig. 2F).

\section{Effect on PPDIS}

Thirteen participants completed the 4 operations (Table 1). For each operation, the pretraining PPDIS scores correlated with the participant's experience level accord-
TABLE 2. Procedural checklist for 4 major pediatric neurosurgical operations

Posterior fossa decompression

1. Ensure proper \& safe patient positioning on the operating table.

2. Demonstrate knowledge of optimum skin incision.

3. Perform an appropriate midline or lateral posterior fossa craniotomy (or craniectomy).

4. Decompress the C-1 arch safely w/o spinal trauma.

5. Achieve adequate exposure through purposeful dissection in the correct tissue planes.

6 . Identify all structures correctly.

7. Perform an appropriate corticotomy.

8. Demonstrate safe use of fixed \& handheld retractors.

9. Define the plane between the tumor \& surrounding brain where appropriate.

10. Demonstrate sound judgment in determining the extent of the tumor resection.

11. Resect the tumor/evacuate the hemorrhage using suction.

12. Achieve intracranial hemostasis.

13. Close the posterior fossa craniotomy.

14. Complete a sound wound repair.

Pterional decompressive craniotomy

1. Position patient \& patient's head appropriately \& safely using pediatric fixation methods.

2. Plan \& mark out appropriate frontotemporal scalp flap, including muscle.

3. Demonstrate safe use of powered burrs, perforator, \& craniotomy to elevate bone flap.

4. Open an appropriate dural flap to protect the underlying cortex.

5. Evacuate the clot or proceed to open sylvian fissure, if needed.

6. Ensure hemostasis.

7. Close \& hitch the dura, where necessary.

8. Secure the bone flap precisely using cranial miniplates or other suitable fixation.

9. Close the galea \& skin in layers.

Tapping the fontanelle to obtain a CSF specimen

1. Identify the fontanelle.

2. Prepare appropriate preparation \& draping to create a sterile field.

3. Demonstrate percutaneous needle \& syringe access to CSF space w/ a 3-way tap.

4. Remove $5 \mathrm{ml}$ of dead space.

5. Remove definitive CSF specimen w/ appropriate volume.

6. Withdraw needle \& apply dressing.

EVD

1. Correct patient positioning.

2. Correct positioning of frontal burr hole.

3. Incise \& reflect the skin, galea, \& pericranium.

4. Demonstrate correct use of a hand brace or high-speed burr.

5. Perform cruciate opening of dura.

6. Puncture cortical surface \& offer to coagulate.

7. Cannulate frontal horn atraumatically using a stilette \& catheter technique. 
» CONTINUED FROM PAGE 331

TABLE 2. Procedural checklist for 4 major pediatric neurosurgical operations

\begin{tabular}{l}
\hline EVD (continued) \\
\hline 8. Ensure free flow of CSF. \\
9. Close galea \& skin. \\
\hline 10. Secure \& dress the EVD exit point. \\
11. Connect the EVD using aseptic technique.
\end{tabular}

The technical stages of each procedure listed are adapted for pediatric equivalent procedures from the current Intercollegiate Surgical Curriculum Programme guidance v9.6. https://www.iscp.ac.uk/Default.aspx. @The Royal College of Surgeons of England, 2006-2015. Adapted with permission.

ingly (Fig. 3). The loss of this correlation after training on babyMARTYN (Fig. 3) reflects the increase in PPDIS for all groups, except participants who rated pretraining with a score of 5 (the post-certificate of completion of training $[\mathrm{CCT}]$ trainee and consultant).

Significant improvements in PPDIS were seen for evacuation of a posterior fossa lesion, pterional craniotomy, tapping of the fontanelle, and EVD insertion $(\mathrm{p}=0.004)$.

\section{Face and Content Validity}

All participants $(n=13)$ said they that they would recommend babyMARTYN and that is was a useful addition to training. The reported performance of the model appearance (visual realism) and tissue handling (tactile realism) is presented as the median Likert scale results $(1=$ unrealistic, $5=$ highly realistic) (Fig. 4).

Free text comments on babyMARTYN's realism and performance as a training model are summarized in Table 3 .

\section{Discussion}

\section{Feasibility and Validation of BabyMARTYN}

We describe the development and potential uses of babyMARTYN, which is a recent pediatric neurosurgical collaborative project with the Royal College of Surgeons of England designed to supplement current neurosurgical training. This project builds on the previous development of MARTYN (the adult neurosurgery training head). ${ }^{3}$ We assessed 4 main neurosurgical procedures commonly performed on infants to test the model's feasibility and validity as a training tool.

We demonstrated face validity, with feedback about the model's tissue handling and appearance having high median scores and bone handling considered the most realistic. The main points for development include the potential for further softening the brain and integrating pressure into the ventricular system. All users reported that babyMARTYN was useful and that they would recommend the model to other trainees, thereby demonstrating content validity. Finally, the model was able to differentiate between junior and senior trainees, with the greatest increase in PPDIS occurring in the least experienced individuals.

Each operation was performed in concordance with
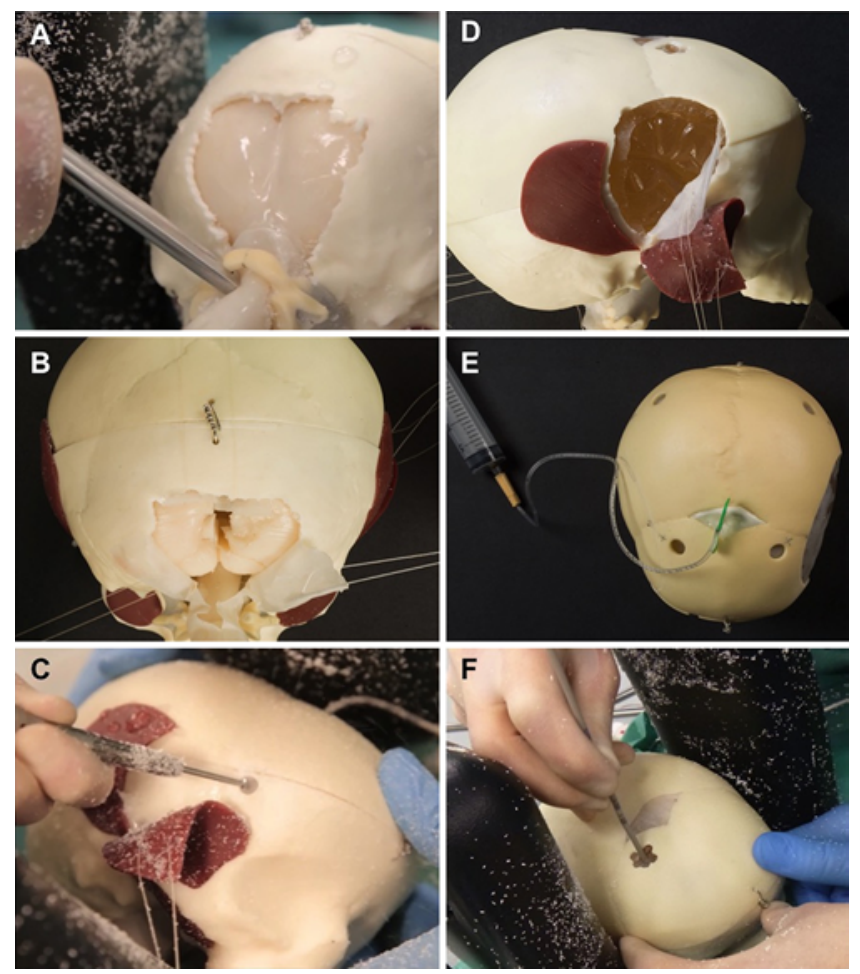

FIG. 2. Operative stages for the 4 main procedures. A: Decompression of the C-1 arch. B: Posterior fossa decompression and clot evacuation. C: High-speed drilling of burr holes for pterional craniotomy. D: Pterional craniotomy. E: Tapping of the fontanelle to obtain a CSF specimen. F: EVD insertion. Panels B, E, and F courtesy of the Royal College of Surgeons of England. Figure is available in color online only.

the procedural checklist based on current guidance found in the current UK curriculum (https://www.iscp.ac.uk/ Default.aspx). The main benefit of the model is that each key stage of each operation was performed. The ability of babyMARTYN to be tested against a curriculum-based checklist demonstrates the model's potential as a training and assessment tool.

\section{Synthetic Training Models in Pediatric Neurosurgery}

There are currently very few pediatric training models in pediatric neurosurgery. One training model, ROWENA (Realistic Operative Workstation for Educating Neurosurgical Apprentices, DELTA Surgical), can be used for the training of pediatric neurosurgical procedures and, of note, is compatible with ultrasound. ${ }^{1}$ However, it is not yet available for infants. Spine simulator models have also been developed to address the unique obstacles encountered in the pediatric spine. ${ }^{7}$ One highly specific pediatric simulator, ASPEN (Anatomical Simulator for Pediatric Neurosurgery, Pro Delphus), includes an anatomical model for craniosynostosis in which biparietal remodeling for scaphocephaly correction can be practiced. ${ }^{2}$ Three-dimensional printing has led to some exciting advancements, and models for pediatric cerebrovascular procedures (both surgical and endovascular) have been found to be feasible and may enable patient-specific models to be produced. ${ }^{15}$ The use of such models resulted in a reduced operative time and pro- 

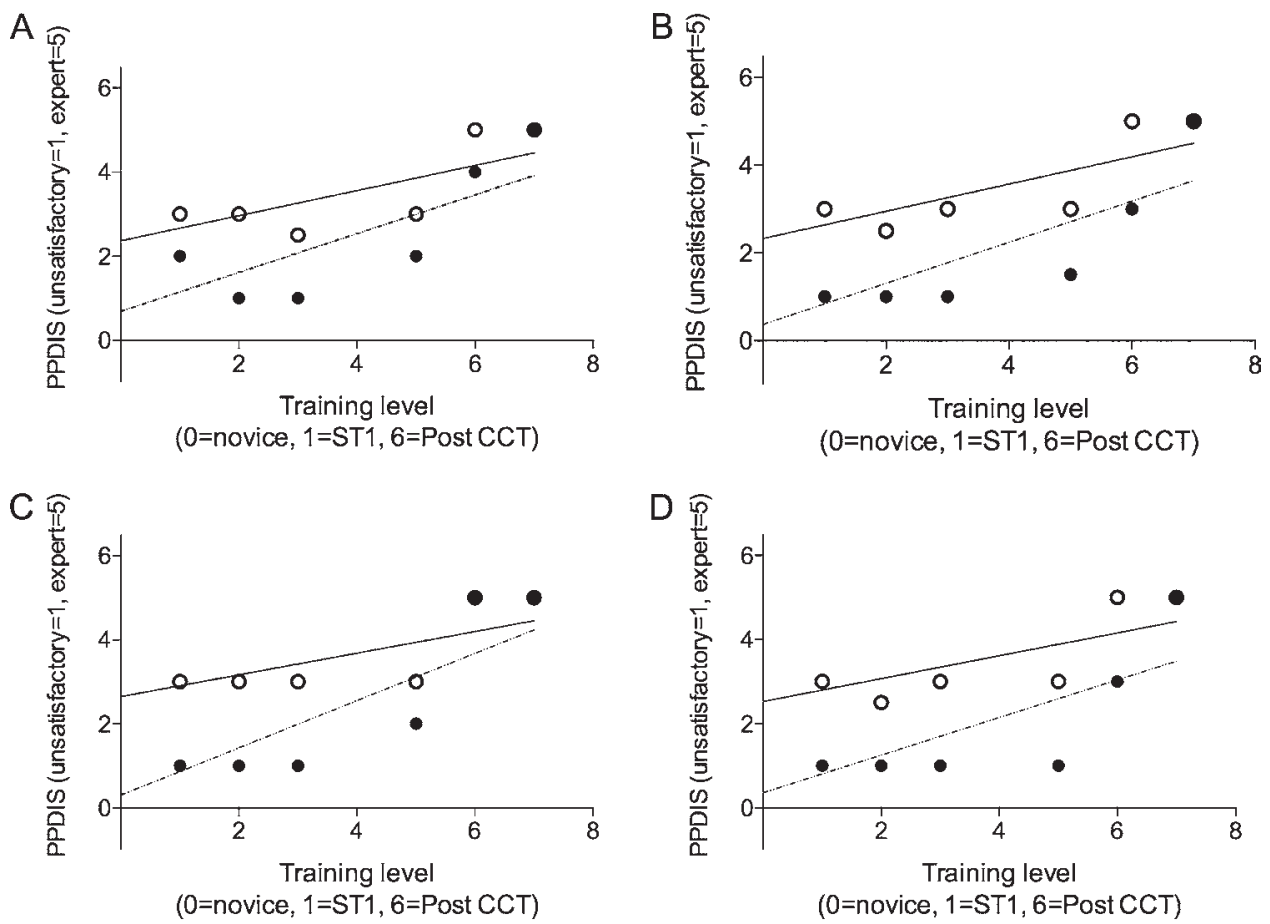

-...... Before - 0 After

FIG. 3. Regression analysis of pretraining PPDIS (self-rated ability) scores with the experience level (specialist trainee [ST] level) before and after training on babyMARTYN for each procedure. A: Evacuation of a posterior fossa lesion (before $\mathrm{R}^{2}=0.58$; after $R^{2}=0.46$ ). B: Pterional craniotomy (before $R^{2}=0.74$; after $R^{2}=0.49$ ). C: Tapping of the fontanelle (before $R^{2}=0.65$; after $R^{2}=$ 0.53). $D$ : EVD insertion (before $R^{2}=0.59$; after $R^{2}=0.49$ ).

vided highly accurate representations of patient anatomy. ${ }^{15}$ This proof-of-concept study shows that babyMARTYN can be used for a range of operations and has good tactile and visual realism. AdultMARTYN has already been shown to be an effective training tool. ${ }^{3}$

\section{Benefits of Synthetic Models to Patient Safety}

BabyMARTYN was developed to fill a niche as a synthetic infant model and has a number of potential benefits for patient safety and neurosurgical trainees. Like many simulation models, babyMARTYN addresses the patient safety issues inherent to a trainee performing a procedure for the first time. This is particularly crucial in infants as their anatomy is unique and presents novel challenges to the neurosurgical trainee. One obvious difference between adult and pediatric neurosurgery is skull thickness. Learning and recognizing the tactile differences during drilling would clearly be more safely learned using a high-fidelity model that removes any unnecessary risk to a child. Practicing on a model removes the immediate risk to the patient while enabling the development of transferable operative skills. ${ }^{13}$ Evidence from other fields, such as anesthesiology, demonstrates that simulator-based training can result in improved patient safety. ${ }^{11}$

\section{Benefits of Synthetic Models to Training}

Before receiving a CCT, neurosurgery trainees must be deemed competent to perform emergency pediatric neurosurgery. There are a number of benefits of using synthetic models to facilitate achieving this competency. Models are not subject to the Human Tissue Act and therefore can be used in various environments (in a teaching room, in

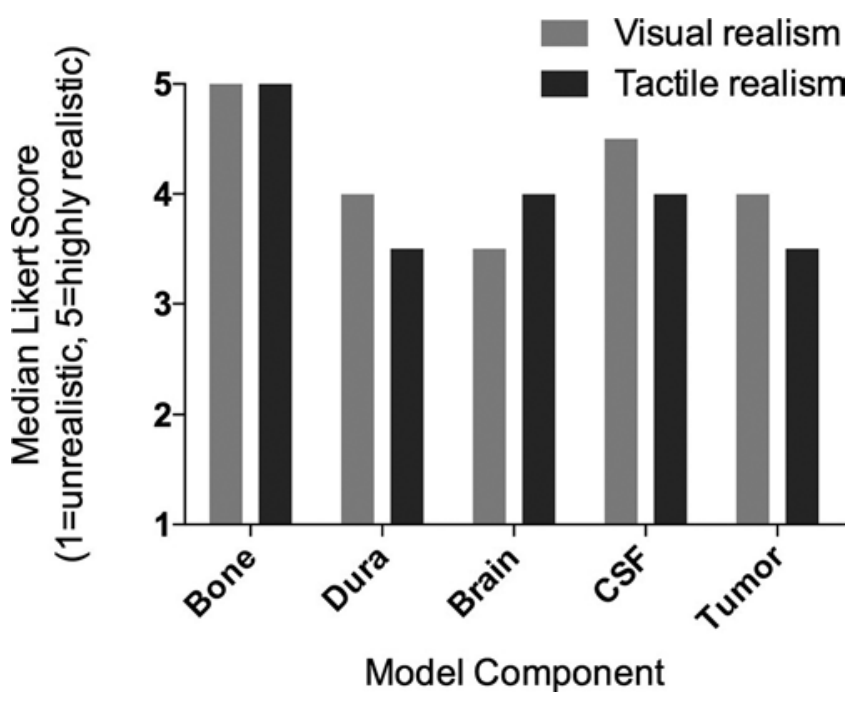

FIG. 4. Face validity assessment of babyMARTYN for visual realism and tactile realism using Likert scale-based results ( $n=13$ responses). 
TABLE 3. Free text explanations

\begin{tabular}{|c|c|}
\hline $\begin{array}{l}\text { Part of the } \\
\text { Model }\end{array}$ & Description \\
\hline Overall & $\begin{array}{l}\text { The model incorporated bone (cranium w/ fontanelle } \\
\text { \& cervical vertebra with the C-1 arch), temporalis } \\
\text { muscle, dura mater, detailed brain parenchyma of } \\
\text { cortex \& cerebellum (with integrated hematoma), } \\
\text { spinal cord, \& ventricles containing CSF. }\end{array}$ \\
\hline Bone & $\begin{array}{l}\text { The response of the synthetic bone to both high-speed } \\
\text { drills \& Kerrison punches was deemed to be ac- } \\
\text { curate in both tactile \& visual realism. The collection } \\
\text { of bone "snow" was realistic \& required regular wash } \\
\text { from the assistant. }\end{array}$ \\
\hline $\begin{array}{l}\text { Dura \& tem- } \\
\text { poralis }\end{array}$ & $\begin{array}{l}\text { Both dura mater \& temporalis muscle also acted like } \\
\text { real tissue being cut \& retracted w/ hitch sutures. }\end{array}$ \\
\hline Brain & $\begin{array}{l}\text { The brain's sulci \& gyri were accurately located. The } \\
\text { posterior fossa hematoma was effectively evacuated } \\
\text { using suction. }\end{array}$ \\
\hline $\begin{array}{l}\text { Ventricles \& } \\
\quad \text { CSF }\end{array}$ & $\begin{array}{l}\text { The CSF was successfully drained via an EVD \& also } \\
\text { by tapping the fontanelle; however, a small amount } \\
\text { of gravity (induced by simply holding the ventricular } \\
\text { catheter below the head) \& negative pressure was } \\
\text { required (via a 5-ml syringe) due to the lack of } \\
\text { increased fluid pressure in the model. The puncture } \\
\text { sensation of entering the ventricle was well repli- } \\
\text { cated \& provided accurate tactile feedback. }\end{array}$ \\
\hline
\end{tabular}

the operating room, or even at home). Unlike cadavers, models do not require special storage, are clean, and do not require sterilization. ${ }^{9}$ Models can be more affordable and readily available than cadavers. Furthermore, models can be custom made for an operation or pathology, even for rare anatomical variations (which is particularly beneficial in pediatrics). BabyMARTYN was developed to include a wide range of pathologies with interchangeable clots and tumors in various locations. Finally, models can be specifically made to simulate each key stage of an operation. Models such as this could enable the practice of basic skills common to pediatric neurosurgery, thus reducing the learning curve and enabling faster achievement of CCT-required competencies. ${ }^{10}$

\section{Surgical Curriculum and Simulation}

Simulation is now integrated formally within the training curriculum in the UK, and there is the potential for models to be used for formal assessments, such as directly observed procedural skills (https:/www.iscp.ac.uk/ Default.aspx). Despite having longer training hours than in the UK, the American Council of Graduate Medical Education also encourages integrating simulation teaching into the surgical curriculum. ${ }^{12}$ In this study, we tested our model against a UK curriculum-based procedural checklist (https://www.iscp.ac.uk/Default.aspx). The model tested the key stages of each checklist item and is therefore compatible with the curriculum's "simulated directly observed procedural skills" assessments.

Nontechnical skills for surgeons within the operative environment will eventually be introduced into the cur- riculum. While we predominantly focus on technical skill in this article, there is potential for this model to integrate both technical and nontechnical skill training using the checklists available within the curriculum website (https:// www.iscp.ac.uk/Default.aspx). ${ }^{4}$ A range of immersive scenarios that tests communication, the ability to cope under pressure, and difficult scenarios could be developed to complement the model. Such scenarios could also be used to train both the individual surgeon and the pediatric team in the operating room.

\section{See One, Simulate One, Do One}

The current "see one, do one, teach one" approach to training is becoming outdated and less acceptable. ${ }^{9}$ An alternative approach might be a "see one, simulate one, do one" method in which the trainee surgeon can perform every step of an operation in sequence on a high-fidelity model in an operating room prior to the surgery. Time permitting, the simulation could even be performed just prior to the real operation, giving confidence to both the trainer and the trainee. As described by Rehder et al., it is essentially giving the trainee a practice swing before hitting the ball. ${ }^{12}$

\section{Challenges and Limitations}

Anecdotally, there are concerns that such training models may distract from clinical teaching in the operating room; however, there is no evidence to support such concerns. On the contrary, while it should not distract from excellence in clinical training, there is plenty of evidence to suggest that simulation can be a valuable adjunct to complement surgical training. ${ }^{13,14}$

\section{Future Studies and Developments}

The stages that cannot currently be assessed with babyMARTYN are hemostasis and skin closure, as the model currently has neither vasculature nor skin. However, such skills are not novel or specific to pediatric neurosurgery. This issue is also present in other training models and methods, such as cadaveric dissection. Skin is currently being integrated into newer prototypes.

Cerebrovascular structures have been developed (albeit for adultMARTYN) and may also be integrated into future prototypes. ${ }^{3}$

\section{Conclusions}

BabyMARTYN is a collaborative pediatric neurosurgical model designed to supplement current pediatric training. Preliminary trials of the model have demonstrated its feasibility in all 4 operations. This pilot trial of the model demonstrates its potential as a training model. BabyMARTYN should be used as an adjunct to current training to enable rehearsal and even assessment of various operations in line with curriculum standards.

\section{References}

1. Ashpole RD: Introducing Rowena: a simulator for neurosurgical training? Ann R Coll Surg Engl 97 Suppl:299-301, 2015 
2. Coelho G, Warf B, Lyra M, Zanon N: Anatomical pediatric model for craniosynostosis surgical training. Childs Nerv Syst 30:2009-2014, 2014

3. Craven C, Baxter D, Cooke M, Carline L, Alberti SJ, Beard J, et al: Development of a modelled anatomical replica for training young neurosurgeons. Br J Neurosurg 28:707-712, 2014

4. Issenberg SB, McGaghie WC, Petrusa ER, Lee Gordon D, Scalese RJ: Features and uses of high-fidelity medical simulations that lead to effective learning: a BEME systematic review. Med Teach 27:10-28, 2005

5. Kirkman MA, Ahmed M, Albert AF, Wilson MH, Nandi D, Sevdalis N: The use of simulation in neurosurgical education and training. A systematic review. J Neurosurg 121:228 246, 2014

6. Marcus H, Vakharia V, Kirkman MA, Murphy M, Nandi D: Practice makes perfect? The role of simulation-based deliberate practice and script-based mental rehearsal in the acquisition and maintenance of operative neurosurgical skills. Neurosurgery 72 (Suppl 1):124-130, 2013

7. Mattei TA, Frank C, Bailey J, Lesle E, Macuk A, Lesniak $\mathrm{M}$, et al: Design of a synthetic simulator for pediatric lumbar spine pathologies. J Neurosurg Pediatr 12:192-201, 2013

8. Milburn JA, Khera G, Hornby ST, Malone PS, Fitzgerald JE: Introduction, availability and role of simulation in surgical education and training: review of current evidence and recommendations from the Association of Surgeons in Training Int J Surg 10:393-398, 2012

9. Murphy M: Surgical simulation: the way forward or a waste of time? Ann R Coll Surg Engl 95 Suppl:289, 2013

10. Patel AD, Gallagher AG, Nicholson WJ, Cates CU: Learning curves and reliability measures for virtual reality simulation in the performance assessment of carotid angiography. J Am Coll Cardiol 47:1796-1802, 2006

11. Rall M, Dieckmann P: Safety culture and crisis resource management in airway management: general principles to enhance patient safety in critical airway situations. Best Pract Res Clin Anaesthesiol 19:539-557, 2005
12. Rehder R, Abd-El-Barr M, Hooten K, Weinstock P, Madsen JR, Cohen AR: The role of simulation in neurosurgery. Childs Nerv Syst 32:43-54, 2016

13. Sturm LP, Windsor JA, Cosman PH, Cregan P, Hewett PJ, Maddern GJ: A systematic review of skills transfer after surgical simulation training. Ann Surg 248:166-179, 2008

14. Sutherland LM, Middleton PF, Anthony A, Hamdorf J, Cregan $\mathrm{P}$, Scott D, et al: Surgical simulation: a systematic review. Ann Surg 243:291-300, 2006

15. Weinstock P, Prabhu SP, Flynn K, Orbach DB, Smith E: Optimizing cerebrovascular surgical and endovascular procedures in children via personalized 3D printing. J Neurosurg Pediatr 16:584-589, 2015

\section{Disclosures}

Mr. Cooke: employee of the Royal College of Surgeons. Dr. Murphy: financial relationship with Murphy and Luis Consultants.

\section{Author Contributions}

Conception and design: Craven, Cooke, Rangeley, Murphy. Acquisition of data: all authors. Analysis and interpretation of data: Craven, Murphy. Drafting the article: Craven. Critically revising the article: Rangeley, Murphy. Reviewed submitted version of manuscript: Craven, Murphy. Approved the final version of the manuscript on behalf of all authors: Craven. Statistical analysis: Craven. Administrative/technical/material support: Cooke, Alberti. Study supervision: Murphy. Model development: Rangeley.

\section{Correspondence}

Claudia L. Craven, Victor Horsley Department of Neurosurgery, National Hospital for Neurology and Neurosurgery, Queen Square, London WC1N 3BG, United Kingdom. email: claudia. craven@gmail.com. 